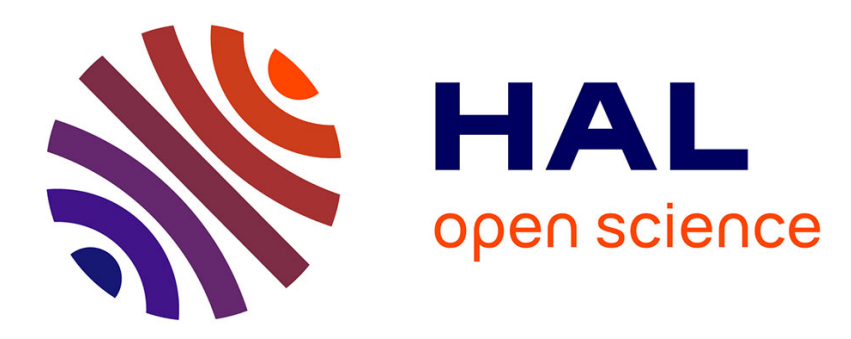

\title{
Nouvelles technologies de développement et inégalités Nord-Sud
}

\author{
Marine Al Dahdah
}

\section{To cite this version:}

Marine Al Dahdah. Nouvelles technologies de développement et inégalités Nord-Sud. Inégalités en perspectives, pp.91-105, 2019, 10.17184/eac.1622 . hal-02520030

\section{HAL Id: hal-02520030 \\ https://hal.science/hal-02520030}

Submitted on 2 Apr 2020

HAL is a multi-disciplinary open access archive for the deposit and dissemination of scientific research documents, whether they are published or not. The documents may come from teaching and research institutions in France or abroad, or from public or private research centers.
L'archive ouverte pluridisciplinaire HAL, est destinée au dépôt et à la diffusion de documents scientifiques de niveau recherche, publiés ou non, émanant des établissements d'enseignement et de recherche français ou étrangers, des laboratoires publics ou privés. 


\title{
Nouvelles technologies de développement et inégalités Nord-Sud
}

Marine Al Dahdah

IFRIS - Cermes3 (CNRS, Paris-Descartes, EHESS, Inserm)

\begin{abstract}
Résumé : Les technologies de l'information et de la communication (TIC) jouent aujourd'hui un rôle de plus en plus central dans les programmes de développement. En 2015, l'organe des Nations Unies chargé des questions de développement et du commerce international, la CNUCED, a rendu public son rapport sur le « développement numérique » et demandé de positionner les TIC au cœur des nouveaux objectifs du développement (UNCTAD 2015). Le positionnement central des TIC dans les programmes internationaux de développement post-2015 est réaffirmé la même année, lors de la définition des « Principes pour le développement numérique » par un groupe d'acteurs issus d'organisations internationales et non gouvernementales. En 2016, le rapport annuel de la Banque mondiale sur le développement dans le monde s'intitule Les Dividendes du numérique et vient confirmer le rôle majeur assigné aux technologies numériques comme levier de développement (World Bank, 2016). Les programmes de santé par téléphone portable - appelés mSanté ou mHealth - sont une forme d'expérimentation de ce développement numérique. À partir de l'exemple d'un dispositif de suivi maternel par téléphone portable déployé au Ghana et en Inde, ce chapitre propose d'explorer un nouveau modèle qui lie le développement économique et social avec l'expansion du téléphone portable et de ses produits. Le recours aux technologies mobiles a introduit dans ces nouveaux programmes de développement des acteurs autrefois étrangers à ce champ et issus en majorité du secteur privé des TIC. Les alliances constituées pour ces programmes se caractérisent par une présence hégémonique d'acteurs privés du Nord, qui financent et déploient des outils techniques en s'appuyant, pour leur mise en œuvre au niveau communautaire, sur les fonctionnaires des services de santé du Sud. La question des rapports Nord-Sud dans les programmes de développement se voit réactualisée à travers ces coopérations technologiques particulières. Ces programmes globaux, encore peu étudiés, constituent une clé de reconfiguration des programmes de développement. En décryptant les inégalités Nord-Sud que ces nouvelles collaborations technologiques impliquent, ce chapitre identifie les logiques hégémoniques qui portent la mSanté.
\end{abstract}

Mots-clés : téléphone portable, numérique, santé, Inde, Ghana

Les technologies de l'information et de la communication (TIC) jouent aujourd'hui un rôle de plus en plus central dans les programmes de développement. En 2015, l'organe des Nations Unies chargé des questions de développement et du commerce international, la CNUCED (Conférence des Nations Unies sur le commerce et le développement) a rendu public son rapport sur le développement numérique et demandé de positionner les TIC au cour des nouveaux objectifs du développement (UNCTAD, 2015, p. 19). Le positionnement central des TIC dans les programmes de développement post-2015 est réaffirmé la même année, lors de la définition des « Principes pour le développe- 
ment numérique ${ }^{1} \gg$ par un groupe d'acteurs issus d'organisations internationales et non gouvernementales ${ }^{2}$. En 2016, le rapport annuel de la Banque mondiale sur le développement dans le monde, Les Dividendes du numérique, et vient confirmer le rôle majeur assigné aux technologies numériques comme levier de développement (World Bank, 2016). Les programmes de santé par téléphone portable - appelés mSanté ou mHealth - sont une forme d'expérimentation de ce développement numérique.

À partir de l'exemple d'un dispositif de suivi maternel par téléphone portable déployé au Ghana et en Inde, ce texte propose d'explorer un nouveau modèle qui lie le développement économique et social avec l'expansion du téléphone portable. Le recours aux technologies mobiles a introduit dans ces nouveaux programmes de développement des acteurs autrefois étrangers à ce champ et issus en majorité du secteur privé des TIC. Les alliances constituées pour ces programmes se caractérisent par une présence hégémonique d'acteurs privés du Nord, qui financent et déploient des outils techniques en s'appuyant, pour leur mise en œuvre au niveau communautaire, sur les fonctionnaires des services de santé de pays dits du $\mathrm{Sud}^{3}$. La question des rapports Nord-Sud dans les programmes de développement se voit réactualisée à travers ces coopérations technologiques particulières. Ces programmes globaux, encore peu étudiés, constituent une clé de reconfiguration des programmes de développement. En décryptant les inégalités Nord-Sud que ces nouvelles collaborations technologiques impliquent, ce texte identifie les logiques impérialistes qui portent la mSanté.

Il mobilise une littérature variée croisant les apports du champ des « Sciences, techniques et sociétés » (STS) sur les technologies comme dispositifs sociaux-techniques, les études des sciences de l'information et de la communication sur les TIC appliquées au développement, enfin le regard des études postcoloniales sur les inégalités NordSud à l'œuvre dans des artefacts techniques. Il s'appuie sur une matière empirique collectée entre 2014 et 2016 au Ghana et en Inde, et mobilise principalement les entretiens réalisés auprès des acteurs responsables de la mise en œuvre de programmes de mSanté dans ces deux pays et ceux directement impliqués dans notre cas d'étude : Motech, le Mobile Technology for Community Health project. Motech a été lancé au Ghana en 2010 par la Fondation Grameen avec le soutien financier de la Fondation Bill et Melinda Gates. L'objectif de Motech est d'améliorer la santé maternelle et infantile en milieu rural dans les pays en développement en accompagnant les femmes durant leur grossesse et jusqu'au premier anniversaire du nouveau-né. Ce projet combine des modules d'information de santé et des messages d'alertes pour les femmes et les professionnels de santé communautaire sous forme de messages vocaux et de SMS. Il repose sur un système d'identification et de traçage des femmes inscrites, de collecte de leurs données de santé et d'analyse de celles-ci à l'aide de formulaires

1. Principles for Digital Development : http ://digitalprinciples.org/

2. Liste des participants : la Fondation Bill et Melinda Gates; la Fondation Omidiyar; l'Agence suédoise de développement international (SIDA); le Fonds des Nations Unies pour l'enfance (Unicef); le Programme des Nations Unies pour le développement (PNUD); UN Global Pulse; le Fonds des Nations Unies pour la population (FNUAP) ; le Haut commissariat des Nations unies pour les réfugiés (UNHCR) ; le Bureau de la coordination des affaires humanitaires des Nations Unies (OCHA); ONU Femmes; la Banque mondiale; le Programme alimentaire mondial (PAM), l'Agence américaine pour le développement international (USaid) ; le département d'État américain, et l'Organisation mondiale de la santé (OMS).

3. On recourra également dans ce texte au vocable « les Suds» pour désigner les pays économiquement « en développement» ou «émergents ». Le vocable «les Nords » sera utilisé pour désigner les pays dits du Nord, c'est-à-dire économiquement « développés ». 
et de rapports. Le but de Motech est de devenir une plateforme « globale » utilisée dans le monde entier afin de soutenir et d'améliorer la qualité et l'accessibilité de l'information de santé et du soin (Grameen Foundation, 2012). À partir de 2012, le projet Motech a été déployé en Inde (État du Bihar) sur le modèle de l'expérience ghanéenne ${ }^{4}$.

La première partie de cet article explore les fondements idéologiques, les mouvements et les acteurs internationaux qui légitiment et promeuvent la technologie mobile et les TIC comme des nouvelles technologies de développement. Elle interroge aussi le « solutionnisme technologique » à l'œuvre dans ces dispositifs; une idéologie qui présente la technologie comme la solution permettant de résoudre les problèmes du monde. La deuxième partie de l'article est consacrée à l'analyse de l'impact de ces développements sur les inégalités Nord-Sud et aux mécanismes de cloisonnement, de protection, de propriété et de coûts qui prolongent des logiques de domination.

\section{Le téléphone portable, une solution de développement?}

Afin de comprendre l'émergence du développement numérique, il est d'abord nécessaire de se tourner vers les pays riches et la « révolution connectée » à laquelle ils ont participé au cours des deux dernières décennies grâce à l'apparition d'un nouveau réseau de communication - l'Internet - et d'un nouveau terminal pour y accéder - le téléphone mobile.

\subsection{La révolution connectée}

Avec la création des premiers sites et du premier moteur de recherche Internet en 1993, la technologie Internet, auparavant secrète et développée à des fins militaires, devient un nouveau média de communication (Abbate, 1999 : 17). En vingt ans, le nombre de ses utilisateurs croît de manière phénoménale pour atteindre 3 milliards fin 2014 (ITU, 2014). L'accès à Internet et les échanges numériques à travers la messagerie électronique, les listes de diffusion, forums, blogs, réseaux sociaux et autres applications populaires du Web ont révolutionné la façon dont les gens vivent et travaillent (Rainie, 2012). L'Internet et les technologies numériques conduisent l'humanité à une nouvelle société connectée, la société en réseau, longuement décrite par Castells. D'après lui, les TIC jouent un rôle aussi fondamental dans la transformation sociale en cours que la machine à vapeur, l'électricité ou les énergies fossiles dans la révolution industrielle. Et cette révolution s'est intensifiée dans les vingt dernières années, grâce aux technologies mobiles (Castells, 2010).

Le premier appel considéré comme émanant d'un téléphone portable utilisant la technologie cellulaire date de 1973, mais l'émergence des lignes mobiles de téléphone

4. Une centaine d'entretiens individuels ont été réalisés avec des professionnels d'institutions impliquées dans la mSanté au Ghana et en Inde : ministères, agences publiques de santé, organismes des Nations Unies, ONG, entrepreneurs du mobile, agences digitales, opérateurs téléphoniques, fondations privées... Sur cette centaine d'entretiens, une quarantaine concernait des acteurs directement impliqués dans la mise en œuvre du projet Motech. Une enquête qualitative dédiée au dispositif Motech a également été conduite dans deux districts du Ghana en 2014 et deux districts de l'État du Bihar en 2015. 35 administrateurs du projet Motech, 50 travailleurs de santé communautaire, une vingtaine de gestionnaires chargés de superviser les travailleurs de santé et environ 200 femmes enrôlés dans le programme ont été interrogés lors d'entretiens de groupes ou en face en face, conduits en anglais ou en langue locale (Fanté et Hindi) avec l'aide d'un assistant de recherche local. 
comme technologie de communication de masse aura lieu deux décennies plus tard. En 1991, le lancement du réseau Global System for Mobile Communication (GSM) marque le décollage de la technologie avec 16 millions d'usagers mobiles dans le monde, un nombre qui s'accroît ensuite de manière exponentielle. La barre des 100 millions d'usagers du mobile est franchie en 1996, et celle du milliard en 2002 (Brown et al., 2002). Mais c'est la rencontre entre Internet et le mobile, avec l'amélioration des terminaux et les réseaux $2 \mathrm{G}$ et $3 \mathrm{G}$ au début des années 2000 qui va démultiplier l'intérêt pour ce terminal. Par sa simplicité d'utilisation, le mobile devient un nouveau terminal d'accès au Web et joue un rôle central dans la révolution numérique (ibid.). De plus, il aura un impact bien plus grand dans les pays en développement qui ne possédaient pas les infrastructures nécessaires pour une connectivité fixe (Castells, 2010). Ainsi, depuis la Silicon Valley, de nombreux entrepreneurs des TIC se tournent vers les pays en développement et participent à un mouvement apparu dans les années 1990, à une idéologie du développement qui s'appuie sur les TIC : l'« information and communication technologies for development »(ICT4D), rebaptisé très récemment « digital development » ou développement numérique.

\subsection{Les TIC pour mettre fin à la pauvreté}

Dès son apparition après la Seconde Guerre mondiale, l'aide au développement était envisagée en termes d'assistance technique et de modernisation des pays moins avancés (Kleine et Unwin, 2009). Réseaux d'électrification, systèmes hydrauliques, pompes, machines industrielles, matériel agricole, véhicules et routes ont constitué et constituent toujours des outils et des moyens d'assistance technique au développement. Les TIC représentent des formes plus récentes de technologies pour le développement et le téléphone mobile, parmi elles, constitue une technologie particulièrement mise en avant. Là où les routes, l'électricité ou encore l'eau n'arrivent pas, le téléphone mobile et Internet prolifèrent. L'idée selon laquelle les TIC, et particulièrement le mobile, touchent plus de gens dans le monde que les infrastructures de base est associée à la promesse d'une nouvelle connexion pour les communautés et populations isolées et (donc) pauvres (Sachs, 2008). Le manque de lignes fixes, le faible coût des infrastructures et des terminaux nécessaires à la connectivité mobile ont permis la diffusion massive du téléphone portable dans les Suds (Katz, 2008). Présenté comme un objet à la fois accessible, « égalitaire » et propice au développement des peuples, le téléphone portable se substitue à un ensemble d'outils difficiles d'accès dans les pays les plus pauvres et devient le terminal principal, voire unique, de connexion à Internet, permettant alors de réaliser toutes les promesses du Web et devenant un dispositif central de réduction de la fracture numérique. Ainsi, par sa plus grande accessibilité, le téléphone mobile, terminal multi facettes, donne accès à des moyens de communication, à des outils technologiques précieux (appareil photo, caméra, enregistreur vocal, calculatrice, organiseur, boîte mail, navigateur Internet, etc.) et à des services mobiles de mEducation, mBanking et mSanté pour remplacer des services scolaires, bancaires ou sanitaires jugés défaillants par les promoteurs du développement numérique (GSMA, 2013, p. 4).

En outre, le téléphone mobile représenterait une clé du développement économique des pays les plus pauvres. D'après la Banque Mondiale et le programme de dévelop- 
pement des Nation-Unies (UNDP), le lien entre développement des réseaux mobiles et croissance économique d'un pays n'est plus à démontrer. Une augmentation de $10 \%$ des usagers de téléphones mobiles entraînerait une augmentation de 0,6 \% du PIB dans les pays développés et de $0,8 \%$ du PIB dans les pays en développement; la croissance des réseaux mobiles dans un pays serait associée à une « augmentation significative »des investissements étrangers directs dans ce pays (UNDP, 2012). Certains 《 techno-optimistes » suggèrent même que les technologies mobiles et Internet permettraient aux pays en développement de 《sauter » des étapes et d'atteindre des niveaux plus élevés de productivité en passant directement à une « économie de la connaissance » (Unwin, 2009).

On observe depuis le début du siècle une littérature croissante, provenant d'institutions et d'acteurs du développement, de consultants ou d'industriels qui cherchent à améliorer l'usage de ces technologies à des fins de développement. Les historiens du développement citent le rapport sur le développement mondial de 1998 de la Banque mondiale intitulé Knowledge for Development comme point de départ de ce mouvement (Heeks, 2010). Cette littérature évalue des dispositifs nouveaux, suggère des améliorations et rapporte des expériences et des «bonnes pratiques 》 ou 《lessons learned ». Elle suit la rhétorique « castellienne » de la révolution connectée et s'en sert comme justification pour ces programmes de développement d'un nouveau type. Ces programmes placent au centre de leur action les TIC, ces outils qui, en leur donnant accès à l'information et à la connaissance, aident les pauvres à se « connecter à leur potentiel », comme nous l'explique le slogan de la fondation Grameen, un des acteurs centraux de Motech ${ }^{5}$. Les promoteurs du développement numérique avancent qu'en diffusant les TIC et en réduisant ainsi la fracture informationnelle dont sont victimes les plus pauvres, la croissance économique s'accélérera, l'agriculture et l'industrie seront plus productives, les services publics plus efficients, la compétitivité des pays en développement sera renforcée et de nombreuses vies seront sauvées (GSMA et McKinsey\& Company 2013; Nilsen et al., 2012; Vital Wave Consulting et mHealth Alliance, 2009 ; Vital Wave Consulting et al., 2009).

Pour ses promoteurs, le téléphone mobile constitue donc une technologie de développement efficace, appliquée par les acteurs de ce champ aux différents grands domaines qui les occupent : la santé, l'éducation, l'agriculture, l'entrepreneuriat solidaire. Et la mSanté rencontre un succès plus important que tous les autres domaines du développement numérique et représente plus des deux tiers des services mobiles pour le développement (GSMA, 2013). Cette prolifération s'explique par des transformations récentes de la santé internationale qui favorisent l'émergence de ces dispositifs socio-techniques. Les dynamiques de globalisation et de marchandisation associées à la «santé globale » justifient et encouragent la propagation des dispositifs techniques comme la mSanté dans les Suds (Atlani-Duault et Vidal, 2013; Geissler, 2015). La mSanté constitue ainsi un laboratoire du développement numérique, un test grandeur nature qui permettrait de solidifier et de crédibiliser des nouvelles techniques numériques de développement.

5. «Connect the world's poor to their potential», site Internet de la Fondation Grameen, consulté le $12 / 02 / 2016$. 


\section{Les limites du « solutionnisme technologique »}

Reformuler les situations sociales complexes et les réduire à des problèmes finis, transparents et objectivables qui peuvent être formulés et résolus de manière évidente et calculable constitue une philosophie centrale de la mSanté, résumée ici par Bill Gates, son premier financeur, sur le site de sa fondation : «To turn caring into action, we need to see a problem, find a solution, and deliver impact ${ }^{6}$. » Cette façon d'envisager les problèmes est qualifiée de « solutionnisme»par Morozov (2013, p. 30). Cet essayiste, critique à l'encontre de la Silicon Valley, s'est attaqué au « solutionnisme technologique » et plus particulièrement à sa version numérique, cette idéologie du Web qui affirme pouvoir résoudre tous les problèmes du monde en un clic. Les acteurs du développement numérique proposent une vision des technologies et de leur pouvoir très proche de celle offerte par les « solutionnistes » de l'Internet. Gates (Microsoft), Schmidt (Google) ou Zuckerberg (Facebook) constituent des figures emblématiques de ce «solutionnisme » et sont des entrepreneurs actifs du développement numérique. Pour ces acteurs influents, les technologies numériques semblent en effet être devenues une réponse systématique aux questions sociales. La connectivité mobile constitue le nouveau cheval de bataille des « solutionnistes » pour pouvoir atteindre les populations non connectées. Présenté comme plus simple à utiliser, plus personnel et plus accessible financièrement qu'un ordinateur, le téléphone mobile devient le terminal pertinent pour diffuser les bienfaits d'Internet partout dans le monde. Cette idéologie qui porte les dispositifs de mSanté véhicule les mêmes aspirations et les mêmes présupposés que ceux mobilisés par de nombreux technologues auparavant. On retrouve dans le développement numérique les discours classiques sur la neutralité des techniques, leur bénéfice évident pour l'humanité et leur séparation du politique qui ont été remis en cause par quantité de chercheurs auparavant (Bijker, 1997 ; Bijker et al., 2012 ; Jasanoff, 2006 ; MacKenzie et Wajcman, 1999). Les techniques étudiées par ces chercheurs, qu'il s'agisse de ponts, de vélos, de néons ou de systèmes photovoltaïques sont imprégnées d'enjeux politiques et sociaux qui conditionnent et/ou contredisent leurs usages et leurs trajectoires (Akrich et al., 2006 ; Bijker et Law, 1992; Winner, 1980). Certains auteurs se sont précisément penchés sur les limites de ces technologies présentées comme des solutions efficaces aux problèmes de développement, notamment à travers l'étude des réseaux hydrauliques (Rottenburg, 2009) ou électriques (Akrich, 1987) déployés dans des pays en développement. Ces auteurs montrent les enjeux multiples qui « empêchent » la translation ou intégration fluide de ces technologies au contexte des pays dits des Suds; et ils révèlent les multiples facteurs sociaux, économiques et politiques qui viennent perturber les scénarios d'usages, les utilisations et les trajectoires des dispositifs techniques.

Dans la lignée de ces travaux, nous souhaitons ici porter un regard critique sur le « solutionnisme » à l'œuvre dans la mSanté. Les TIC sont souvent présentées comme des outils neutres qui permettront des avancées exceptionnelles pour les pays en développement. Si Internet à ses débuts était rêvé comme un espace libre, neutre, sans dieu ni maître, plusieurs chercheurs se sont consacrés à l'étude de la gouvernance du Net, ses règles, les normes que le réseau impose, les enjeux de pouvoirs très forts qui le

6. Gates, cité dans «Who we are - Bill \& Melinda Gates Foundation » (Qui nous sommes - Bill \& Melinda Gates Foundation), consulté le 25/02/16. 
caractérisent (Abbate, 1999; Brousseau et al., 2012 ; Cardon, 2013; Musiani, 2013). Le mobile n'est pas différent des autres dispositifs socio-techniques étudiés par ces universitaires. Il n'est pas neutre, il incarne des idéologies, des politiques et des décisions particulières. Comme pour bien d'autres technologies, le téléphone mobile évolue et incorpore de nouvelles idées, normes et conceptions qui viennent des industriels, des développeurs, des régulateurs comme des usagers (Oudshoorn et Pinch, 2003).

Plusieurs chercheurs proposent d'ailleurs une analyse critique de cette vision du développement par les TIC (Berg et Toussaint, 2003; Pieterse, 2010a; Wade, 2002 ; Ya'u, 2004). Certains dénoncent par exemple le réductionnisme à l'œuvre dans les programmes qui prônent la fin de la fracture numérique dans les pays dits des Suds et un développement économique corrélé avec l'accès au téléphone mobile; ils appellent à la prudence face à une vision technicisée de la fracture numérique et expliquent que celle-ci ne se réduit pas à l'accès technologique, qu'il s'agit d'un problème plus complexe avec des enjeux politiques et socio-économiques (Pieterse, 2010a). Comme Akrich ou Rottenburg l'ont montré pour les réseaux hydrauliques ou électriques, les TIC nécessitent des prérequis technologiques, politiques et des infrastructures institutionnelles autant que techniques pour pouvoir fonctionner dans les pays en développement. Wade met en avant l'idée que réduire la fracture numérique entre Nord et Sud signifierait diminuer les écarts de revenus alors même qu'ils ne cessent de se creuser (Wade, 2002). Pieterse ajoute qu'une part majeure de l'accroissement des inégalités dans le monde est en fait liée à un différentiel de plus en plus important dans les compétences techniques associées aux TIC, et c'est précisément sur la maîtrise et la production des TIC que les écarts se creusent (Pieterse, 2010b). Enfin, Ya'u explique que la fracture numérique ne fait qu'augmenter malgré les programmes déployés pour tenter de la réduire; il dénonce également l'impérialisme technologique des pays dits du Nord qui possèdent les canaux d'information et diffusent des contenus sur lesquels les pays dits des Suds n'ont aucun pouvoir (Ya'u, 2004). Ainsi, pour ces différents auteurs, présenter les technologies numériques comme solution aux inégalités du monde apparaît antinomique.

La deuxième partie de cet article souhaite illustrer les limites de ce « solutionnisme » appliqué à des programmes de développement à travers l'exemple de Motech qui révèle et transforme de nombreuses inégalités sur ses terrains d'application. Nous proposons d'examiner plus précisément les inégalités Nord-Sud reconduites ou renforcées par le dispositif technique et de montrer comment le système de santé local censé bénéficier de cette innovation technique est en fait affaibli par cette approche particulière du développement.

\section{Impérialisme technologique et inégalités Nord-Sud}

Si, au XIX ${ }^{\mathrm{e}}$ siècle, le terme d'impérialisme renvoyait à une forme de gouvernement qui dépend d'un empire, au $\mathrm{XX}^{\mathrm{e}}$ siècle le terme évolue pour décrire une forme de gouvernement associée à l'économie capitaliste. Comme l'explique Raymond Williams, si l'impérialisme est envisagé comme un système économique, les pratiques extractives et le contrôle des marchés perdurent bien après la chute des empires coloniaux (Williams, 1985). Les termes de « nouvel ou néo-impérialisme » (Harvey, 2003) ou 
« impérialisme américain » (Smith, 2003) sont mobilisés pour décrire les nouvelles formes d'hégémonie et d'asservissement économique qui s'exercent aujourd'hui. Cette partie de notre texte mobilise la notion d' «impérialisme technologique » parce qu'elle fait référence au temps long, à l'histoire des sciences et des techniques, à des continuités historiques entre des régimes de pouvoir différents qui utilisent la science et les techniques à des fins de domination (Petitjean et al., 1992). La notion d'impérialisme permet d'inscrire les formes de domination actuelles dans une continuité et de montrer que, s'il revêt des habits nouveaux, le régime de domination à l'œuvre dans les dispositifs étudiés au Ghana et en Inde repose sur des pratiques d'exploitation et d'asservissement anciennes (Brukum Kwaku, 1985; Arnold, 2000).

Qui possède les technologies proposées par ces programmes de développement? Les pays du Sud bénéficiaires ont-ils la possibilité d'en modifier la forme et le contenu? Peuvent-ils maintenir eux-mêmes le dispositif sans aide extérieure? L'expérience de Motech permet de répondre à ces questions et de comprendre plus précisément où se jouent les inégalités Nord-Sud lorsque des dispositifs mobiles pour le développement sont déployés dans les Suds. Nous proposons donc d'explorer quatre points sur lesquels s'exercent des inégalités Nord-Sud associées aux plateformes mobiles : les savoirs et savoir-faire, les enjeux de propriété, l'interopérabilité des systèmes, enfin les coûts des dispositifs.

\section{Les capacités techniques des Suds}

Développer et gérer des systèmes d'information nécessite des connaissances spécialisées et un savoir-faire particulier, mais les acteurs du développement numérique estiment que les pays dans lesquels sont déployés les dispositifs techniques ne possèdent pas ces savoirs et ne peuvent donc pas les gérer ni les maintenir sans l'aide des pays dits développés (Sim et Philip, 2008). Motech constitue une excellente illustration de cette croyance qui maintient une inégalité de savoirs entre les Nords et les Suds.

Lorsque le ministère de la santé ghanéen (GHS) collabore à des projets de télésanté, deux de ses directions sont particulièrement concernées : celle qui est en charge de la politique, du contrôle et de l'évaluation des établissements et la direction des systèmes d'information et de communication. La première a été impliquée dès la conception du projet Motech en 2010 pour des raisons logistiques. En effet, elle gère les structures et les personnels de santé indispensables pour déployer le dispositif Motech sur le terrain. Mais ce rôle crucial du GHS n'est plus une évidence dès lors qu'on se penche sur la partie technique du dispositif. En effet, le GHS ne possède pas la plateforme technique Motech, n'a pas accès à la base des données alimentée par ses propres équipes et ne peut utiliser Motech sans un accord avec la Fondation Grameen qui gère la plateforme. Cette mise à l'écart du ministère pour la partie technique de Motech serait liée pour certains acteurs à un déficit de « compétences techniques ». En effet, la Fondation Grameen met en doute les capacités du ministère à administrer la plateforme Motech, se positionnant ainsi comme le seul technicien apte à gérer Motech.

Pourtant, lors de l'enquête de terrain au Ghana, plusieurs professionnels extérieurs au ministère et à Grameen ayant travaillé pour Motech avançaient que, si la direction des systèmes d'information et de communication du ministère avait été intégrée au 
projet, elle aurait eu les compétences pour gérer la plateforme Motech. En excluant les techniciens du ministère, la Fondation Grameen ne laissait pas la possibilité aux ingénieurs ghanéens d'acquérir le savoir-faire associé à cette plateforme spécifique et ne s'appuyait pas sur leurs connaissances du terrain. Lors de nos enquêtes, nous avons rencontré les deux directions du ministère et assisté à des formations sur des applications de mSanté développées par la direction des systèmes d'information et de communication du ministère. Si les équipes du ministère ne maîtrisaient pas le code exact de Motech, elles développaient et administraient néanmoins des systèmes mobiles d'information de santé similaires; elles pouvaient donc potentiellement se former au code Motech afin de pouvoir gérer cette plateforme. Le cas indien est similaire. Alors même que l'Inde fait figure de géant des technologies numériques, le ministère indien ne gère pas la plateforme Motech. Comme nous l'ont expliqué les fonctionnaires du ministère, les spécificités des serveurs demandés justifient que les Fondations américaines gardent la main sur Motech.

Ce jugement sur les capacités des pays du Sud à gérer des systèmes d'information est omniprésent dans les projets de développement numérique. Il révèle une inégalité majeure entre les maîtres d'œuvre et les récipiendaires de ces dispositifs. Il illustre la volonté de fermer l'objet technique en proposant des configurations trop spécifiques pour pouvoir être gérées et adaptées aux configurations locales. Comme l'explique Wade, ces programmes technologiques enferment les pays du Sud dans une nouvelle dépendance vis-à-vis des pays du Nord en passant par des normes et des régimes technologiques développés par et pour ces derniers (Wade 2002). Les capacités techniques des pays du Sud constituent effectivement un véritable enjeu pour l'incorporation pérenne de ces dispositifs techniques. Mais, dans le cas de Motech, le transfert de compétences ne s'est pas effectué pour d'autres raisons, car dès 2013 le Ghana et l'Inde possédaient les ressources humaines et technologiques pour gérer une plateforme comme Motech. L'étude de Motech révèle des problèmes de propriété de la plateforme, d'interopérabilité et de coûts importants qui ont été plus déterminants encore que les « capacités techniques des Suds» dans la trajectoire du dispositif.

\section{Propriété et «Open source »}

La «plateforme » ou «suite»Motech, comme elle est appelée par ses concepteurs, désigne le système informatique qui rassemble les programmes nécessaires au fonctionnement des applications qui constituent Motech Ghana et Motech Inde. Les questions autour de la propriété et des droits d'utilisation de cette plateforme Motech appellent des réponses contrastées. D'après la fondation Grameen, la question des droits de propriété n'a pas lieu d'être, puisque Motech est une plateforme sous licence Open source et donc libre d'accès, comme l'explique cette employée de Grameen : «If you want to use Motech, it's open source, you just go on the Internet, you download it and look at the code and see what it does, if you need any help then you come to us. » [Si vous voulez utiliser Motech, c'est Open source, il suffit juste d'aller sur Internet, de le télécharger et de regarder le code, et si vous avez besoin d'aide vous venez nous demander.] (Entretien A., Grameen Foundation, 07/14.) Mais, pour le ministère de la santé ghanéen, la fondation Grameen est seule propriétaire de la plateforme : «Motech platform is owned by the Grameen foundation. The data belongs to the Grameen 
foundation right now and the platform is used by them for other countries such as India. » [La plateforme Motech appartient à la fondation Grameen. Les données appartiennent à la fondation et ils utilisent la plateforme dans d'autres pays comme l'Inde par exemple.] (Entretien M., GHS, 05/14.)

Si la licence Open source est accessible à tous, pour pouvoir utiliser la plateforme il ne s'agit pas de s'acquitter de droits de propriété, mais d'acquérir des compétences et des savoirs techniques indispensables pour pouvoir utiliser le code html, paramétrer et faire fonctionner tous les composants de Motech. Si l'Open source est souvent avancé comme outil pour compenser les inégalités d'accès aux logiciels, il reste un artefact technique difficilement transférable (Sim et Philip, 2008). Il n'y a donc pas de «propriétaire » de Motech avec un copyright établi, mais, dans les faits, seule la Fondation Grameen peut utiliser la plateforme. Les deux autres applications ghanéennes de mSanté - No Yawa et CCH - qui utilisaient Motech en 2014 sont entièrement gérées par la Fondation Grameen. Les autres partenaires qui portent ces projets - DKT International, Marie Stopes, Concern Worldwide - n'ont pas accès à la plateforme et à la base de données associée. Il en est de même en Inde, le ministère indien de la Santé et la fondation de la BBC qui gèrent des projets utilisant Motech doivent passer par Grameen pour toute utilisation et évolution de la plateforme.

Lorsque nous avons évoqué les différents dysfonctionnements de Motech avec l'équipe technique de la fondation Grameen, nos interlocuteurs nous ont expliqué qu'ils étaient liés à une maintenance difficile de la plateforme en raison du turnover des équipes techniques et de l'apprentissage du code Open source de Motech, qui semblait plus complexe que ne le laissait penser l'interviewée citée quelques paragraphes ci-dessus. La fondation Grameen reconnaît les difficultés d'utilisation du code Motech et a mis en place des dispositifs de formation pour ses équipes entrantes, mais n'a pour l'instant pas mis en place ce transfert de compétences pour les autres partenaires du projet qui, à terme, devaient pourtant récupérer le dispositif, comme les ministères de la santé ghanéen ou indien par exemple. Le « déficit de compétences techniques » pour gérer Motech se retrouve donc chez tous les autres partenaires de Motech, qu'ils soient ghanéens, indiens ou américains. Il s'agit en fait d'une forme de protectionnisme de la part de la Fondation qui a une expertise à vendre et souhaite rester dans le «business ». L'Open source n'est en rien un gage d'accessibilité ou une garantie de transfert technologique : sans transfert de savoirs et de savoir-faire associés au logiciel, celui-ci reste aussi impénétrable qu'une version propriétaire.

\section{Interopérabilité et cloisonnement numérique}

Pour Motech comme pour d'autres projets, les acteurs locaux n'ont pas accès à la partie technique du dispositif, ils ne peuvent modifier ce canal ni même accéder aux données collectées par leurs soins. La plateforme Motech ne communique avec aucune autre plateforme de données. Aussi, les données collectées par les travailleurs de santé pour Motech ne sont pas « interfacées » avec les systèmes nationaux d'information de santé, elles sont stockées et gérées par la Fondation Grameen de manière cloisonnée. Les travailleurs de santé doivent donc saisir des données de santé pour Motech, puis à nouveau dans le système d'information de santé centralisé. Cette opération de 
double saisie est systématiquement effectuée, car le registre Motech, trop simplifié, ne comprend pas l'ensemble des informations que le système central exige sur la santé maternelle et infantile. On observe donc deux systèmes de collecte de données parallèles qui ne communiquent pas et une double saisie d'informations, source de perte de temps pour les professionnels sur le terrain. Cette absence d'interopérabilité et la faible prise en compte des besoins d'information des systèmes d'information de santé nationaux ont été dénoncées par plusieurs acteurs impliqués dans Motech, au Ghana et en Inde.

Dans ces deux pays, les équipes techniques et les technologies locales sont systématiquement pointées du doigt comme étant en retard, fermées, peu adaptables, moins compétentes et responsables de l'absence d'interopérabilité entre les systèmes. Si, au Ghana le système d'information de santé centralisé n'existait pas encore au lancement de Motech en 2010, l'Inde quant à elle possédait déjà un dispositif d'information de santé national au moment de l'arrivée de Motech en 2012 sur lequel les équipes Motech auraient pu travailler pour une meilleure communication entre les deux systèmes. Mais les standards des plateformes mobiles en évolution constante se font loin d'Accra ou de Delhi et les normes de la Silicon Valley s'imposent globalement et sans condition, sous peine de ne pas être référencées par Google ou Apple et, donc, de ne pas exister dans le monde « connecté ».

\section{Technologies «Low-cost»}

La question du coût de développement et de maintenance des dispositifs constitue un autre sujet problématique. Une plateforme comme Motech nécessite des dépenses multiples sur lesquelles les acteurs impliqués ne veulent jamais indiquer de prix. Lorsque nous avons demandé aux acteurs de détailler les coûts techniques de la plateforme, ils ne donnaient jamais de montant clair. Tout ce qui concerne les coûts et les dépenses du projet Motech demeure opaque, en Inde comme au Ghana, et beaucoup de transferts ne sont pas chiffrés puisqu'il s'agit parfois de transferts de compétences ou de déductions de prestations. Pour le projet national en Inde, la fondation Gates n'a pas d'échange financier ou de contrat avec le ministère de la santé indien. Pourtant, d'après le ministère, une équipe de gestion du projet Motech a été installée pendant trois ans en son sein aux frais de la fondation Gates et celle-ci a également pris en charge tous les coûts associés au lancement du dispositif national, en plus du développement de la plateforme. Loin d'être négligeables, ces coûts sont invisibles et ne relèvent d'aucune bourse officielle. En Inde comme au Ghana, il est impossible d'avoir une visibilité sur les revenus générés par les applications payantes de Motech (Kilkari et Mobile Midwife MTN). Interrogés sur les revenus générés par ces applications commerciales, nos interlocuteurs nous ont expliqué que l'objectif n'était pas de faire de l'argent et que le dispositif coûtait de toute façon bien plus cher que ce qu'il rapportait. Mais ils étaient incapables de nous dire précisément quels étaient ces coûts. Les seuls coûts transparents sont ainsi ceux de la connectivité mobile annoncés par les opérateurs téléphoniques partenaires des dispositifs que l'on propose d'analyser maintenant. 
Si l'on se penche sur Motech au Ghana, le seul coût de la connectivité, c'est-à-dire de l'accès au réseau mobile et au transfert des données sur ce réseau, constitue déjà un frein majeur à la pérennité du projet Motech. Le marché des télécommunications est dominé par les firmes multinationales des pays du Nord. En Afrique subsaharienne par exemple, à l'exception de MTN (sud-africain) et Airtel (indien), tous les groupes télécoms appartiennent à des pays développés (Ya'u, 2004). Le Ghana ne possède pas de compagnie nationale de télécommunications, le secteur est entièrement privatisé. Il paye donc le prix fort comme beaucoup d'autres pays du Sud pour pouvoir accéder aux services de téléphonie mobile. Aussi, pour mettre à disposition des femmes enceintes des messages vocaux gratuits, l'État ghanéen devrait a minima s'acquitter des coûts de connectivité facturés par les opérateurs téléphoniques. Or, financer la connectivité de Motech au Ghana constitue un coût exorbitant pour l'État.

En effet, le seul coût d'envoi des messages aux 9000 femmes enrôlées dans le district pilote de Gomoa représente une dépense mensuelle de près de 4000 euros (assumée par la Fondation Gates); un coût que le ministère ne peut prendre en charge. Ainsi, dans le cadre d'une extension nationale de Motech, le seul coût d'envoi des messages vocaux aux femmes ciblées s'élèverait à plus de 220 millions d'euros par an. Cela signifie que, sur les trente euros par an que le gouvernement ghanéen consacre à la santé d'une Ghanéenne, huit euros supplémentaires seraient nécessaires pour envoyer un message hebdomadaire Motech, soit presque un quart de la somme totale. Pour l'État ghanéen, le dispositif Motech n'est pas une solution économique, il ne permet pas de réduire les dépenses générales de santé - et l'on ne s'appuie ici que sur les coûts de connectivité du dispositif, qui constituent un seul des postes de dépenses de la mSanté. Au lieu de réduire les coûts de santé, la promesse de low cost de la mSanté s'inverse et le dispositif Motech entraîne une inflation spectaculaire des coûts de santé pour l'État. Les financements nécessaires au fonctionnement de la mSanté s'avèrent ainsi importants pour des pays dont les ressources financières consacrées à la santé sont limitées. Les coûts des dispositifs de développement numérique comme Motech sont loin d'être transparents et le retour sur investissement est d'autant moins calculable que l'impact de ces dispositifs n'est pas évalué de manière indépendante et claire. De plus, même si les coûts du dispositif Motech pouvaient être absorbés par l'État, l'inscription de ce dispositif au sein du système de santé national poserait des questions majeures en raison de son manque d'interopérabilité et des différents blocages liés à la maîtrise du code source de Motech que nous avons détaillés.

\section{Conclusion}

Les inégalités générées par l'utilisation des technologies numériques dans les pays en développement sont peu étudiées. Le téléphone mobile est avant tout présenté comme un progrès, une innovation économique et sociale, une promesse de réussite. Ce texte a montré que le «solutionnisme technologique » qui porte les dispositifs de développement numérique comme Motech tend à masquer les dépendances et les inégalités Nord-Sud que de tels dispositifs technologiques peuvent générer. L'exemple de Motech met en avant quatre mécanismes qui amplifient des inégalités technologiques entre les pays dits du Nord et du Sud. Premièrement, les capacités techniques des Suds sont mises en cause systématiquement et justifient l'absence de transfert des savoirs néces- 
saires au maintien des dispositifs. Deuxièmement, les blocages dans la transmission du code source d'applications qui se disent Open source reconduisent des formes de propriété sur les logiciels défavorables aux Suds. Troisièmement, l'interopérabilité entre les systèmes issus des Nords et ceux déjà en place dans les Suds est empêchée par un cloisonnement numérique lié à une obsolescence technique dictée par des normes issues des Nords. Enfin, les coûts importants des dispositifs - entre autres, les montants facturés par les multinationales des Télécommunications - rendent impossible le financement des dispositifs mobiles par l'État qui ne parvient pas toujours à négocier avec les opérateurs privés. Les enjeux de gouvernance de la santé internationale liés au nouveau modèle de développement numérique et les arbitrages à prendre sont d'autant plus cruciaux que les États du Sud consacrent à la santé une part de leur budget qui stagne, voire diminue, chaque année. Ainsi, l'exemple de Motech vient montrer la façon dont des priorités globales viennent éclipser des enjeux nationaux et mettre à mal les systèmes publics de santé des pays du Sud en leur imposant des technologies sur lesquelles ils n'ont pas de maîtrise ${ }^{7}$.

\section{Références}

Abbate J. (1999), Inventing the Internet, Cambridge, Mass, MIT Press (Inside technology).

Akrich M. (1987), « Comment décrire les objets techniques?», Techniques \& Culture, p. 49-64, https ://hal shs.archives-ouvertes.fr/halshs-00005830/.

Akrich M., Callon M., Latour B. et Centre de sociologie de l'innovation (Paris) (2006), Sociologie de la traduction : textes fondateurs, Paris, Mines Paris, les Presses.

Arnold D ; (2000), Science, technology and medicine in colonial India, Cambridge University Press, vol. 5 Atlani-Duault L. et Vidal L. (2013), La santé globale, nouveau laboratoire de l'aide internationale?, Paris, Armand Colin.

Berg M. et Toussaint P. (2003), « The mantra of modeling and the forgotten powers of paper : a sociotechnical view on the development of process-oriented ICT in health care », International journal of medical informatics, vol. $69, \mathrm{n}^{\circ} 2$, p. 223-234

Bijker W.E. (1997), Of bicycles, bakelites, and bulbs : toward a theory of sociotechnical change, Cambridge, Mass., MIT Press.

Bijker W.E. et Law J. (1992), Shaping technology/building society : studies in sociotechnical change, Cambridge, Mass., MIT Press.

Bijker W.E., Hughes T.P. et Pinch T.J. (2012), The social construction of technological systems : new directions in the sociology and history of technology, Anniversary ed, Cambridge, Mass, MIT Press.

Brousseau E., Marzouki M. et Méadel C. (2012), Governance, regulations and powers on the Internet, Cambridge; New York, Cambridge University Press.

Brown, B., Green, N. et Harper, R. (dir.) (2002), Wireless world : social and interactional aspects of the mobile age, London; New York, Springer (Computer supported cooperative work).

Brukum Kwaku N. J. (1985), "Afro-European Relations on the Gold Coast, 1791-1844 ». M.A. thesis, Legon, University of Ghana.

Cardon D. (2013), « Politique des algorithmes », Réseaux, vol. 177, n 1, p. 9.

Castells M. (2010), The rise of the network society, 2nd ed., With a new pref, Chichester, West Sussex, Malden, MA, Wiley-Blackwell (The information age : economy, society, and culture).

Geissler, W. (dir.) (2015), Para-states and medical science : making African global health, Durham, Duke University Press (Critical global health).

Grameen Foundation (2012), «MOTECH Lessons Learned ». Grameen Foundation, Motech, $2^{\text {nd }}$ edition.

7. L'auteure a bénéficié d'un financement de l'ANRS pour sa recherche doctorale. 
GSMA (2013), «ANALYSIS Scaling Mobile for Development Harness the opportunity in the developing world ». GSMA Intelligence. https ://gsmaintelligence.com/files/analysis/ ?file=130828-scaling-mobile.pdf \&utmsource=MDI\&utmcampaign =95fa2bd3c5-report-2013-07-26\&utmmedium =email\&utmterm =082a920 fd6a-95fa2bd3c5-300686685.

GSMA et McKinsey\&Company (2013), «mHealth : A new vision for healthcare », http ://www.gsma.com/ connectedliving/wp-content/uploads/2012/03/gsmamckinseymhealthreport.pdf (Consulté le 18 décembre 2017)

Harvey D. (2003), The new imperialism, Oxford; New York : Oxford University Press.

Heeks R. (2010), « Do information and communication technologies (ICTs) contribute to development?», Journal of International Development, vol. 22, $\mathrm{n}^{\circ} 5$, p. 625-640.

International Telecommunication Union (ITU) (2014), World in 2014 : ICT Facts and Figure, Geneva, International Telecommunication Union; http ://www.itu.int/en/ITU-D/Statistics/Documents/facts/ICTFa ctsFigures2014-e.pdf

Jasanoff S. (2006), States of knowledge : the co-production of science and social order, London, Routledge.

Katz J.E. (2008), Handbook of Mobile Communication Studies. Cambridge, Mass. : MIT Press. http ://site .ebrary.com/lib/cbuc/docDetail.action?docID $=10229587$.

Kleine D. et Unwin T. (2009), « Technological Revolution, Evolution and New Dependencies : what's new about ict4d? », Third World Quarterly, vol. 30, n 5, p. 1045-1067.

MacKenzie D.A. et Wajcman J. (1999), The social shaping of technology, 2nd ed, Buckingham (Eng.), Philadelphia, Open University Press.

Morozov E. (2013), To Save Everything, Click Here : Technology, Solutionism, and the Urge to Fix Problems that Don't Exist, Allen Lane.

Musiani F. (2013), « La gouvernance des algorithmes »? https ://adam.hypotheses.org/1791.

Nilsen W., Kumar S., Shar A., Varoquiers C., Wiley T., Riley W.T., Pavel M. et Atienza A.A. (2012) «Advancing the Science of mHealth», Journal of Health Communication, vol. 17, $\mathrm{n}^{\circ}$ sup1, p. 5-10.

Oudshoorn N. et Pinch T.J. (2003), How users matter: the co-construction of users and technologies, Cambridge, Mass, MIT Press (Inside technology).

Petitjean P., Jami C. et Moulin A.-M. (dir.) (1992,) Science and Empires Historical Studies about Scientific Development and European Expansion. Dordrecht : Springer Netherlands, http ://public.eblib.com/cho ice/PublicFullRecord.aspx $? \mathrm{p}=3070741$.

Pieterse J. (2010a), « Digital capitalism and development : The unbearable lightness of ICT4D », in Development Theory : Deconstructions/Reconstructions, London, SAGE Publications Ltd.

Pieterse J. (2010b), Development Theory : Deconstructions/Reconstructions, London, SAGE Publications Ltd.

Rainie H. (2012), Networked : the new social operating system, Cambridge, Mass, MIT Press.

Rottenburg R. (2009), Far-fetched facts : a parable of development aid, Cambridge, Mass, The MIT Press (Inside technology).

Sachs J. (2008), « The digital war on poverty ». The Guardian.

Sim S.E. et Philip K. (2008), Tracing Transnational Flows of IT Knowledge Through Open Exchange of Software Development Know-How, https ://www.ideals.illinois.edu/handle/2142/15075.

Smith N. (2003), American empire : Roosevelt's geographer and the prelude to globalization. California, Univ of California Press, vol. 9

UNCTAD (2015), « Digital Developement Report », Commission on Science and Technology for Development, eighteenth session? http ://unctad.org/en/Pages/MeetingDetails.aspx ?meetingid=606.

UNDP (2012), «Mobile Technologies and empowerment : enhancing human development through participation and innovation ». http ://www.undpegov.org/sites/undpegov.org/files/undpmobiletechnologyprimer. pdf.

Unwin P.T.H. (2009), ICT4D : Information and Communication Technology for Development, Cambridge, Cambridge University Press.

Vital Wave Consulting et mHealth Alliance (2009), « Sizing the Business Potential of mHealth in the Global South ». http ://www.mHealthAlliance.org 
Vital Wave Consulting, UN Foundation et Vodafone Foundation (2009), «mHealth for Development : The Opportunity of Mobile Technology for Healthcare in the Developing World », http ://vitalwave.com/wpcontent/uploads/2015/09/Vital WaveConsulting mHealth6March09 pdf.pdf

Wade R.H. (2002), « Bridging the Digital Divide : New Route to Development or New Form of Dependency? », Global Governance, vol. 8, n 4, p. 443-466

Williams R. (1985), Keywords : a vocabulary of culture and society. Rev. ed., New York, Oxford University Press.

Winner L. (1980), « Do artifacts have politics? », Daedalus, p. 121-136.

World Bank (2016), World Development Report 2016 : Digital Dividends. Washington, DC : World Bank.

World Health Organization (WHO) 2013, World Health Statistics. 2013, Geneva, World Health Organization, http ://www.who.int/gho/publications/worldhealthstatistics/EN WHS2013Part3.pdf?ua=1.

Ya'u Y.Z. (2004), « The new imperialism \& Africa in the global electronic village », Review of African Political Economy, vol. 31, n 99, p. 11-29. 